\title{
Early Third Trimester
}

National Cancer Institute

\section{Source}

National Cancer Institute. Early Third Trimester. NCI Thesaurus. Code C141437.

The period of gestation generally corresponding to the first half of the third trimester. 